\title{
Demonstration of a programmable source of two-photon multiqubit entangled states
}

\author{
Simone Cialdi, ${ }^{1,2}$ Davide Brivio, ${ }^{1}$ and Matteo G. A. Paris ${ }^{1,3}$ \\ ${ }^{1}$ Dipartimento di Fisica dell'Università degli Studi di Milano, I-20133 Milano, Italia \\ ${ }^{2}$ INFN, Sezione di Milano, I-20133 Milano, Italia \\ ${ }^{3}$ CNISM UdR Milano Università, I-20133, Milano, Italia
}

(Received 18 December 2009; published 26 April 2010)

\begin{abstract}
We suggest and demonstrate a novel source of two-photon multipartite entangled states which exploits the transverse spatial structure of spontaneous parametric down-conversion together with a programmable spatial light modulator (SLM). The one-dimensional SLM is used to perform polarization entanglement purification and to realize arbitrary phase gates between polarization and momentum degrees of freedom of photons. We experimentally demonstrate our scheme by generating two-photon three-qubit linear cluster states with high fidelity using a diode laser pump with a limited coherence time and power on the crystal as low as $\sim 2.5 \mathrm{~mW}$.
\end{abstract}

DOI: 10.1103/PhysRevA.81.042322 PACS number(s): 03.67.Bg, 03.67.Mn, 42.50.Dv, 42.50.Ex

\section{INTRODUCTION}

Multiqubit entangled states (e.g., cluster states) are key resources to realize several protocols of quantum-information processing, including measurement-based quantum computation [1-3], quantum communication [4], and quantum error correction [5]. Moreover, earlier work identified applications in advanced fundamental tests of quantum nonlocality [6-9]. Basically, there are two ways to generate multiqubit entangled states (e.g., cluster states). On one hand, one may increase the number of entangled photons [10-13]. On the other hand, one may use different degrees of freedom of the same pair of photons [3,14-16] achieving so-called hyperentanglement. The second method offers a larger robustness against decoherence and nonunit detector efficiency. Four and six multiphoton cluster states have been experimentally created [10-12] as well as two-photon four- [3,14,15,17-25] and six-qubit cluster states [26].

In this paper we suggest and demonstrate a scheme to generate two-photon multipartite entangled states which exploits the transverse spatial structure of spontaneous parametric down-conversion (PDC) and a one-dimensional programable spatial light modulator (SLM) based on a liquid crystal display. These kinds of devices have already been used as pulse shapers for Bell state generation [27], as amplitude modulators for momentum imaging and qudit generation [28] as well as diffractive elements to operate on orbital angular momentum [29]. Here, we employ SLM in an innovative way to realize two-photon multiqubit or multiqudit entangled states and demonstrate its use in the generation of two-photon three-qubit linear cluster states with high fidelity.

The novelty of our setup is twofold. On the one hand, we use the SLM for purification, and this allows us to dramatically decrease the spectral and angular filtering of down-converted photons, which is the method generally used to prevent the degradation of the purity. Moreover, the SLM may be externally controlled, via software, and this makes our method more easily adjustable for the different implementations, compared to purification schemes that involve the use of suitably prepared crystals along the path of the down-converted photons [30]. On the other hand, we fully exploit the properties of the SLM to realize arbitrary phase gates between polarization and momentum degrees of freedom. In this way, we obtain an effective, low-cost, source of two-photon multipartite entanglement using a pump with low power and a limited coherence time.

The paper is structured as follows. In Sec. II we describe our PDC system in some details and illustrate the purification method based on the use of SLM. In Sec. III we address the generation of two-photon multiqubit or multiqudit entangled states and describe our experimental setup, used to demonstrate the generation of two-photon three-qubit linear cluster states with high fidelity. Sec. IV closes the paper with some concluding remarks.

\section{POLARIZATION ENTANGLEMENT AND PURIFICATION}

The first step in our scheme is the generation of polarization entangled states by spontaneous parametric down-conversion (SPDC) in two adjacent BBO crystals oriented with their optical axes aligned in perpendicular planes [31-33]. The state at the output of the two crystals may be written as

$$
\begin{aligned}
|\Phi\rangle= & \frac{1}{\sqrt{2}} \int d \theta d \omega_{s} d \omega_{p} f\left(\omega_{p}, \omega_{s}, \theta\right) A\left(\omega_{p}\right) \\
& \times\left[e^{\imath k_{p}^{o}\left(\omega_{p}\right) L} e^{\imath \phi(\theta)+\imath \phi^{\prime}\left(\theta^{\prime}\right)}\left|H, \theta, \omega_{s}\right\rangle\left|H, \theta^{\prime}, \omega_{p}-\omega_{s}\right\rangle\right. \\
& \left.+e^{\imath k_{2 \|}\left(\omega_{p}, \omega_{s}, \theta\right) L}\left|V, \theta, \omega_{s}\right\rangle\left|V, \theta^{\prime}, \omega_{p}-\omega_{s}\right\rangle\right],
\end{aligned}
$$

where $L$ is the crystals' length and $|P, \theta, \omega\rangle$ denotes a single photon state with polarization $P=H, V$, emitted at angle $\theta$ and frequency $\omega$. The complex amplitude spectrum of the pump laser is $A\left(\omega_{p}\right)$ whereas the down-converted photons are generated with the two photons' spectral and angular amplitude $f\left(\omega_{p}, \omega_{s}, \theta\right)$, as defined in [33]. We call $\overline{\omega_{p}}=\omega_{p}^{0}+$ $\omega_{p}$ and $\overline{\omega_{s}}=\omega_{p}^{0} / 2+\omega_{s}$ the frequencies of the pump laser and of the signal, where $\omega_{p}$ and $\omega_{s}$ are the shift from the central frequencies $\omega_{p}^{0}$ and $\omega_{p}^{0} / 2$. Likewise, signal and idler generation angles are, respectively, $\bar{\theta}=\theta^{0}+\theta$ and $\overline{\theta^{\prime}}=-\theta^{0}+\theta^{\prime}$, with $\theta^{0}$ the central angle and $\theta^{\prime}=-\theta+\gamma \omega_{s}+\gamma^{\prime} \omega_{p}$. Within the spectral width of our pump the dependence on $\omega_{p}$ is negligible and thus we have $\theta^{\prime} \simeq-\theta+\gamma \omega_{s}$, with $\gamma=\partial \theta^{\prime} / \partial \omega_{s}$.

The phase term $k_{p}^{o}\left(\omega_{p}\right) L$ is due to the pump traversing the first crystal before it generates photons in the second one, whereas the term $k_{2 \|}\left(\omega_{p}, \omega_{s}, \theta\right) L$ appears because the photons 
generated in the first crystal must traverse the second one. The perpendicular part $k_{2 \perp}$ disappears for conservation of the transverse momentum, as it is guaranteed by the large pump spot on the crystals $(\sim 1.5 \mathrm{~mm})$. The other phase terms are common and are grouped out. The phase shifts $\phi(\theta)$ and $\phi^{\prime}\left(\theta^{\prime}\right)$ are introduced by a spatial light modulator, respectively, on the signal and on the idler and depend on the generation angles $\bar{\theta}$ and $\bar{\theta}^{\prime}$. These will be discussed in detail in the following. It can be shown numerically that $f\left(\omega_{p}, \omega_{s}, \theta\right) \approx f\left(0, \omega_{s}, \theta\right) \equiv$ $f\left(\omega_{s}, \theta\right)$ for crystals' length $L \lesssim 1 \mathrm{~mm}$. Upon expanding all the contributions to the optical paths to first order and after some algebra we arrive at

$$
\begin{aligned}
|\Phi\rangle= & \frac{1}{\sqrt{2}} \int_{-\Delta \theta / 2}^{\Delta \theta / 2} d \theta \int_{\omega_{s 1}(\theta)}^{\omega_{s 2}(\theta)} d \omega_{s} \\
& \times \int d \omega_{p} f\left(\omega_{s}, \theta\right) A\left(\omega_{p}\right) \cdot\left[\left|H, \theta, \omega_{s}\right\rangle\left|H, \theta^{\prime}, \omega_{p}-\omega_{s}\right\rangle\right. \\
& \left.+e^{\imath \varphi\left(\omega_{p}, \omega_{s}, \theta\right)}\left|V, \theta, \omega_{s}\right\rangle\left|V, \theta^{\prime}, \omega_{p}-\omega_{s}\right\rangle\right]
\end{aligned}
$$

where $\Delta \theta$ is the angular acceptance and $\omega_{s 1,2}=(1 / \gamma)(\theta \mp$ $\Delta \theta / 2)$ are the integration limits for $\omega_{s}$, as determined by $\Delta \theta$ and $\gamma$. The phase function between the $H$ and the $V$ component is given by

$\varphi\left(\omega_{p}, \omega_{s}, \theta\right)=\phi_{0}+\alpha L \omega_{p}+\beta L \omega_{s}-\delta L \theta-\phi(\theta)-\phi^{\prime}\left(\theta^{\prime}\right)$,

where $\phi_{0}$ includes all the zero-order terms of the expansion. The phase term $\alpha L \omega_{p}$ accounts for the delay time between horizontal and vertical down-converted photons. The two subsequent terms rise for conservation of the transverse momentum. The term $\beta L \omega_{s}$ may be understood by considering the signal at the fixed angle $\bar{\theta}$ : For different $\omega_{s}$ the idler sweeps different $\overline{\theta^{\prime}}$ and this means different optical path. Likewise, fixing $\omega_{s}$, a positive variation of $\theta$, corresponds to a negative variation of $\theta^{\prime}$ and this introduces an optical path dependent on $\theta$ (i.e., the phase shift $\delta L \theta$ ). The delay time between the photons may be compensated upon the introduction of a proper combination of birefringent crystals on the pump path, as already demonstrated in [32] (see Fig. 2). Let us now focus attention on the action of the SLM [i.e., on the phase function $\phi(\theta)$ and $\left.\phi^{\prime}\left(\theta^{\prime}\right)\right]$. At first, since $\theta^{\prime} \simeq-\theta+\gamma \omega_{s}$, we note that the choice,

$$
\phi^{\prime}\left(\theta^{\prime}\right)=\beta L \theta^{\prime} / \gamma+\epsilon^{\prime} \quad \phi(\theta)=-\beta L \theta / \gamma+\epsilon,
$$

with $\epsilon^{\prime}+\epsilon=\phi_{0}$ allows one to compensate all the remaining phase terms in $\varphi\left(\omega_{p}, \omega_{s}, \theta\right)$ and to achieve purification of the state. In this way, we may generate polarization entangled states as in Eq. (2) with $\varphi\left(\omega_{p}, \omega_{s}, \theta\right)=0$. Experimentally, we obtain a visibility of about $\sim 0.9$ starting from $\sim 0.42$.

\section{TWO-PHOTON MULTIPARTITE ENTANGLEMENT}

In order to generate multipartite entangled states, and in particular cluster states, purification is just the first step. Here, we propose a technique based on the use of the SLM. We consider the signal and the idler beams divided in $N$ and $M$ subdivisions [see Fig. 1(d)], which individuate different momentum qudits, and write the signal and idler momentum state as

$$
|s\rangle=\sum_{n}^{N} a_{n}|n\rangle_{s}, \quad|i\rangle=\sum_{m}^{M} a_{m}|m\rangle_{i},
$$

with $n=0,1, \ldots, N-1$ and $m=0,1, \ldots, M-1$. The total momentum state is $|\Psi\rangle=|s\rangle \otimes|i\rangle$. This is not an entangled state in the momentum since for a certain signal angle $\theta$, the idler sweeps a wide interval of $\theta^{\prime}$, actually covering all the angular acceptance $\Delta \theta$ due to the broad down-conversion spectrum. The global state is thus given by $|\Phi\rangle \otimes|\Psi\rangle$, where polarization provides two qubits, and the rest of information is encoded onto the momentum degrees of freedom [34-36].

The action of the SLM corresponds to impose a phase shift only on the horizontal component of polarization, leaving the vertical part undisturbed. We exploit this property to add a different constant phase, besides the purification ones $\phi(\theta)$ and $\phi^{\prime}\left(\theta^{\prime}\right)$, for each portion of signal and idler. This corresponds to the action of a set of controlled phase gates $C_{\phi}, \boldsymbol{\phi}=$ $\left\{\phi_{0 i}, \ldots, \phi_{M-1 i}, \phi_{0 s}, \ldots, \phi_{N-1 s}\right\}$ to the state $|\Phi\rangle \otimes|\Psi\rangle$. Using a suitable number of sectors (power of two) one may generate multiqubit entangled states of the form $|\Xi\rangle=C_{\phi}|\Phi\rangle \otimes|\Psi\rangle$.

The simplest example, which we implemented experimentally, is obtained using $M=1$ and $N=2$ (i.e., by dividing the signal beams in two parts exploiting the SLM to apply a phase $\phi$ to only one of them); see Figs. 1(a) and 1(b). This leads to the generation of a two-photon three-qubit entangled state of the form $\frac{1}{2}\left[|000\rangle+|110\rangle+e^{\imath \phi}|001\rangle+|111\rangle\right]$ where, for the first two qubits, $|0\rangle \equiv|H\rangle$ and $|1\rangle \equiv|V\rangle$ whereas the

(a)

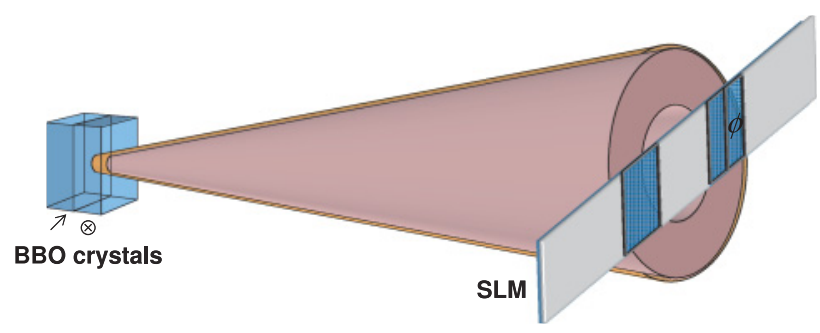

(b)

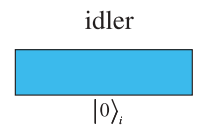

(c)

(d)
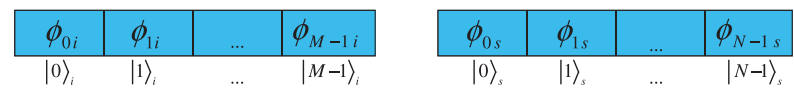

FIG. 1. (Color online) Generation of multipartite entangled or cluster states by the use of SLM. The overall output from SPDC is divided in spatial sections, and a different phase may be imposed to each portion in a programmable way, corresponding to the application of controlled phase gates. Each spatial section includes several pixels. In (b) we report the momentum state in our experimental implementation $|\Psi\rangle=\frac{1}{\sqrt{2}}|0\rangle_{i}\left(|0\rangle_{s}+|1\rangle_{s}\right)$; in (c) the state configuration to achieve $|\Psi\rangle=\frac{1}{2}\left(|0\rangle_{i}+|1\rangle_{i}\right)\left(|0\rangle_{s}+|1\rangle_{s}\right.$ ) (which may become $|\Psi\rangle=\frac{1}{\sqrt{2}}(|0\rangle|1\rangle+|1\rangle|0\rangle)$ upon the use of a spectral filter, bandpass of $10 \mathrm{~nm})$. In (d) the generic configuration leads to the momentum state $|\Psi\rangle=|s\rangle \otimes|i\rangle$. 
third qubit is the signal momentum. For $\phi=\pi$ one obtains a two-photon three-qubit linear cluster state,

$$
\left|C_{3}\right\rangle=\frac{1}{\sqrt{2}}\left[\left|\Phi^{+}\right\rangle|0\rangle-\left|\Phi^{-}\right\rangle|1\rangle\right],
$$

where $\left|\Phi^{ \pm}\right\rangle$are standard Bell states. In order to highlight the power of our method let us consider another example, with four qubits: for $M=N=2$ [see Fig. 1(c)] and applying $\phi_{0 s}=$ $-\phi_{0 i}, \phi_{1 i}=\pi-\phi_{1 s}$ we achieve the four-qubit entangled state,

$$
\begin{aligned}
\left|\Xi_{4}\right\rangle= & \frac{1}{2}\left[\left|\Phi^{+}\right\rangle|00\rangle-\left|\Phi^{-}\right\rangle|11\rangle\right. \\
& \left.+\left|\Delta^{+}\left(\phi_{t}\right)\right\rangle|01\rangle-\left|\Delta^{-}\left(\phi_{t}\right)\right\rangle|10\rangle\right],
\end{aligned}
$$

where $\phi_{t}=\phi_{0 i}+\phi_{1 s}$ and $\left|\Delta^{ \pm}\left(\phi_{t}\right)\right\rangle=\frac{1}{\sqrt{2}}\left[|00\rangle \pm e^{\mp l \phi_{t}}|11\rangle\right]$. We foresee that using a narrower spectral filter for the downconverted photons it is possible to select different regions of the angular distribution in a way that allows us to engineer entanglement also for the momentum degrees of freedom. Using a $10-\mathrm{nm}$ bandpass filter and coupling $\Delta \theta \simeq 1.6 \mathrm{mrad}$ on the momentum channels $|n\rangle_{s},|m\rangle_{i}$, for $N, M=2$, we would have $|\Psi\rangle=\frac{1}{\sqrt{2}}(|0\rangle|1\rangle+|1\rangle|0\rangle)$. In such a way the total state would be the two-photon four-qubit cluster state reported in $[14,15]$.

\section{A. Experiment}

The experimental setup is shown in Fig. 2. The pump derives from a 405-nm cw laser diode (Newport LQC 405-40P). After a half-wave plate (HWP) that rotates its polarization in order to balance the generated state, the pump passes through two BBO crystals that compensate the delay time $\alpha L$ between $|H\rangle$ and $|V\rangle$ generated photons. Two BBO crystals, each cut for type-I down-conversion, stacked back-to-back and oriented with their optical axes aligned in perpendicular planes, are used to generate the polarization entangled state.

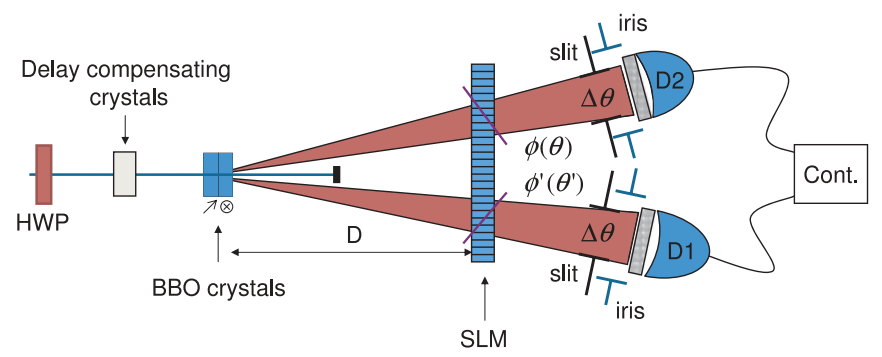

FIG. 2. (Color online) Experimental setup. Polarization entangled states are generated by down-conversion in type-I BBO crystals pumped at $405 \mathrm{~nm}$ by a Newport LQC $405-40 \mathrm{P}$ cw laser diode. Then, a spatial section of the output cones passes through the SLM which (at the same time) i) provides purification of polarization entanglement and ii) introduces a position- (i.e., generation angle) dependent phase shift between the two polarizations. After SLM, there are (on both paths) a slit, an iris, two long-pass filters (cut-on wavelength $=715 \mathrm{~nm}$ ), a coupler with an $1 / e^{2}$ output beam diameter of $7.14 \mathrm{~mm}$, and a multimode optical fiber that directs the photons to the detector. The detectors are homemade single-photon counting modules $(D 1, D 2)$, based on an avalanche photodiode operated in Geiger mode with passive quenching. For tomographic reconstruction we insert a quarter-wave plate, a half-wave plate, and a polarizer and for the optimization of the phase functions only the polarizers.
As shown in Fig. 1(a), a portion of the output cones passes through the SLM, which is a crystal liquid phase mask $(64 \times 10 \mathrm{~mm})$ divided in 640 horizontal pixels, each wide $d=100 \mu \mathrm{m}$ and with the liquid crystal $10-\mu \mathrm{m}$ deep. The SLM is set at a distance $D=500 \mathrm{~mm}$ from the two generating crystals. Driven by a voltage, the liquid crystal orientation in correspondence of a certain pixel changes. The photons with a horizontal polarization feel an extraordinary index of refraction, depending on the orientation, and this introduces a phase shift between the two polarizations. Since each pixel is driven independently we can introduce a phase function dependent on the position on the SLM (i.e., on the generation angle $\theta$ and $\theta^{\prime}$ ).

It is worth noting that the SLM also replaces the birefringent plate used for the optimal generation of photon pairs [31]. After SLM, on the signal and the idler paths, there are a slit, an iris, two long-pass filters (cut-on wavelength = $715 \mathrm{~nm}$ ), a coupler with an $1 / e^{2}$ output beam diameter of $7.14 \mathrm{~mm}$, and a multimode optical fiber that directs the photons to the detector. The detectors are homemade single-photon counting modules $(D 1, D 2)$, based on an avalanche photodiode operated in Geiger mode with passive quenching. For the tomographic reconstruction we insert, on both paths after the iris, a quarter-wave plate, a half-wave plate, and a polarizer. For the optimization of the phase functions (see below) we insert only the polarizers.

Our experimental setup allows us to collect the downconverted photons within a wide spectrum and angular distribution. In order to underline this fact the pump power on the crystals has been intentionally left very low $(2.5 \mathrm{~mW})$ by using an amplitude modulator. To collect as many photons as possible we make the imaging of the pump spot on the crystals ( $\simeq 1.5 \mathrm{~mm}$ ) into the optical fiber's core (diameter of $62.5 \mu \mathrm{m}$ ) using the coupler lenses. Setting the slits at $4 \mathrm{~mm}(\Delta \theta \simeq 6.5$ $\mathrm{mrad}$ ) and the iris with a diameter of $9 \mathrm{~mm}$ we collect up to 100 coincidence counts per second. It is worthwhile to note that such an angular acceptance $\Delta \theta$ acts as a $100-\mathrm{nm}$ bandpass spectral filter for the down-converted photons. In order to purify the state we insert the phase functions,

$$
\phi(x)=a_{2}\left(x-x_{c 2}\right)+b_{2} \quad \phi^{\prime}(x)=a_{1}\left(x-x_{c 1}\right)+b_{1},
$$

where $x$ is the pixel number, $x-x_{c 2}=\frac{D}{d} \theta$ and $x-x_{c 1}=$ $\frac{D}{d} \theta^{\prime}, x_{c 1}$, and $x_{c 2}$ are the central pixels on idler and signal (i.e., the pixels corresponding to the central angles $\overline{\theta^{\prime}}=-\theta^{0}$ and $\left.\bar{\theta}=\theta^{0}\right)$. The values of the parameters $a_{1}, b_{1}, a_{2}$, and $b_{2}$ have been optimized upon inserting two polarizers set at $\alpha_{1}=45^{\circ}$ and $\alpha_{2}=-45^{\circ}$ in front of the couplers and then searching for the minima in the coincidence counts, corresponding to the values of $b_{1,2}$ compensating the constant phase difference $\phi_{0}$ and $a_{1,2}$, removing the angular dependence on $\theta$ and on $\theta^{\prime}$, and, in turn, on $\omega_{s}$. For our configuration, we have $a_{1}=$ $-a_{2}=\beta L d / \gamma D \simeq-0.05, b_{1}+b_{2}=\phi_{0}$.

In Fig. 3(a) we report the coincidence counts on a time window equal to $30 \mathrm{~s}$ as a function of $b_{1}\left(b_{2}\right)$ as blue circles (red squares) with $b_{2}=0\left(b_{1}=0\right)$ and with $a_{1,2}$ set to their optimal values. In Fig. 3(b) we report the coincidence counts on a time window of $30 \mathrm{~s}$ as a function of $a_{1}=-a_{2}$ with $b_{2}=0$ and $b_{1}=\phi_{0}$. The agreement with the theoretical model is excellent. In turn, the purification of the state works as follows: 

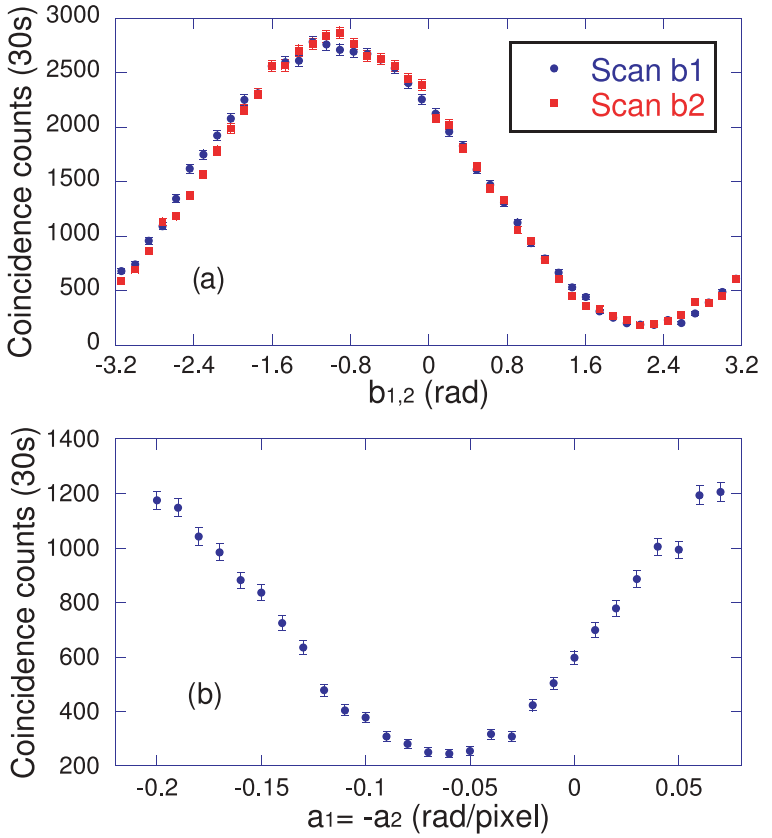

FIG. 3. (Color online) Coincidence counts on a time window of $30 \mathrm{~s}$ with the polarizers in front of the couplers set at $45^{\circ}$ and $-45^{\circ}$. (a) Coincidences as a function of $b_{1}$ (blue) and $b_{2}$ (red) with $b_{2,1}=0$ and optimal $a_{1,2}$; (b) coincidences as a function of $a_{1}=-a_{2}$ with optimal $b_{1}$ and $b_{2}=0$.

Starting from a visibility equal to $0.423 \pm 0.016$ we achieve $0.616 \pm 0.012$ after the delay compensation with the crystals and $0.886 \pm 0.012$ after the spatial compensation with the SLM. Finally, by closing the iris at the same width of the slits we obtain $0.899 \pm 0.008$. Actually, we verified experimentally that variations of the phase in the azimuthal direction have only a minor effect. The residual lack of visibility is in turn due to the low spatial coherence of the pump, which is spatially multimode.

\section{B. State reconstruction}

Upon properly programming the SLM [i.e., by setting $M=1, N=2$, and $\phi=\pi$ as in Fig. 1(b)], our scheme may be set to generate, in ideal conditions, the cluster state $\left|C_{3}\right\rangle$. In order to characterize the output state, denoted by $R_{3}$, and to check the effects of the decoherence processes, we have performed state reconstruction by (polarization) quantum tomography [37,38]. The experimental procedure goes as follows: upon measuring a set of independent two-qubit projectors $P_{\mu}=\left|\psi_{\mu}\right\rangle\left\langle\psi_{\mu}\right|(\mu=1, \ldots, 16)$ corresponding to different combinations of polarizers and phase shifters, the density matrix may be reconstructed as $\varrho=\sum_{\mu} p_{\mu} \Gamma_{\mu}$, where $p_{\mu}=\operatorname{Tr}\left[\varrho P_{\mu}\right]$ are the probabilities of getting a count when measuring $P_{\mu}$ and $\Gamma_{\mu}$ is the corresponding dual basis (i.e., the set of operators satisfying $\operatorname{Tr}\left[P_{\mu} \Gamma_{\nu}\right]=\delta_{\mu \nu}$ [39]). Of course, in the experimental reconstruction, the probabilities $p_{\mu}$ are substituted by their experimental samples (i.e., the frequencies of counts obtained when measuring $P_{\mu}$ ). In order to minimize the effects of fluctuations and avoid nonphysical results, we use maximum-likelihood reconstruction of two-qubit states $[37,38]$.
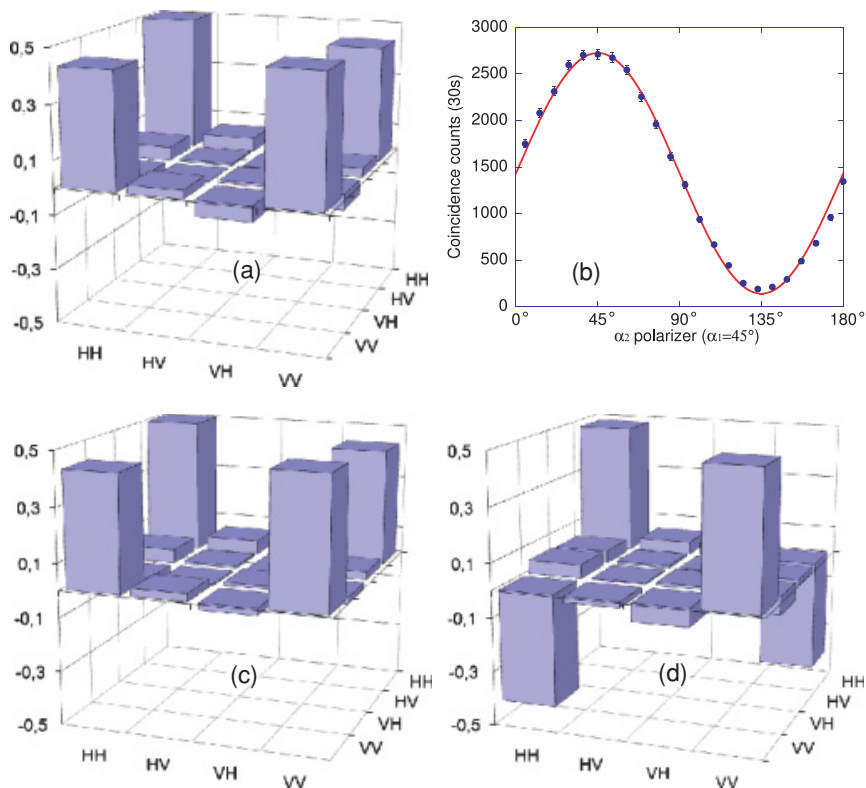

FIG. 4. (Color online) Characterization of the output state. In (a) we report the tomographic reconstruction (real part) of the global purified polarization entangled state prior to the action of the phase gate, whereas in (b) we show the corresponding visibility curve and the fit with the curve $\cos ^{2}\left(\alpha_{2}-45^{\circ}\right)$ (solid line). In (c) and (d) we report the tomographic reconstructions (real part) of the reduced states $\varrho_{0}$ and $\varrho_{1}$.

At first we reconstruct the purified state prior to the action of the SLM phase gate (i.e., without addressing the momentum qubit). Then, we reconstruct the two reduced states $\varrho_{j}=\frac{1}{p_{j}} \operatorname{Tr}_{3}\left[|j\rangle_{s s}\langle j| R_{3}\right]$ obtained by measuring the momentum qubit after the phase gate. This is obtained by moving the slit on the signal to select the corresponding portion of the beam. Results are summarized in Fig. 4.

As is apparent from the plots, our scheme provides a reliable generation of the target states. Fidelity of the purified polarization state is about $F \simeq 0.90 \pm 0.01$, whereas fidelities of the conditional states $F_{0}=\left\langle\Phi^{+}\left|\varrho_{0}\right| \Phi^{+}\right\rangle$and $F_{1}=\left\langle\Phi^{-}\left|\varrho_{1}\right| \Phi^{-}\right\rangle$are given by $F_{0}=0.92 \pm 0.01$ and $F_{1}=$ $0.90 \pm 0.01$, respectively. In order to achieve this precision, we have employed a long acquisition time $(\sim 60 \mathrm{~s})$, thus, also demonstrating the overall stability of our scheme. We also report the visibility of the state prior to the action of the SLM phase gate, which confirms the entanglement purification process [40].

\section{CONCLUSIONS}

We have suggested and implemented a scheme for the generation of two-photon multipartite entangled states. In our device, a programmable spatial light modulator acts on different spatial sections of the overall down-conversion output and provides polarization entanglement purification as well as arbitrary phase gates between polarization and momentum qubits. It should also be mentioned that measurements on the momentum qubits benefit from our configuration. In fact, addressing momentum is equivalent to selecting portions of 
the signal (idler) beam and then making them interact, say by a beam splitter and other linear optical elements, to perform arbitrary momentum measurements. In our scheme this may be implemented in a compact form since the portions of the beam are quite close to each other, and we may work with the beam splitter at non-normal incidence. Overall, our scheme represents an effective, low-cost, source of two-photon multiqubit or qudit entanglement. We foresee applications in one-way quantum computation and quantum error correction.

\section{ACKNOWLEDGMENTS}

The authors thank I. Boscolo for encouragement and support.
[1] R. Raussendorf and H. J. Briegel, Phys. Rev. Lett. 86, 5188 (2001).

[2] M. A. Nielsen, Rep. Math. Phys. 57, 147 (2006).

[3] G. Vallone, E. Pomarico, F. DeMartini, and P. Mataloni, Phys. Rev. Lett. 100, 160502 (2008).

[4] R. Cleve, D. Gottesman, and H. K. Lo, Phys. Rev. Lett. 83, 648 (1999).

[5] D. Schlingemann, and R. F. Werner, Phys. Rev. A 65, 012308 (2001).

[6] N. D. Mermin, Phys. Rev. Lett. 65, 1838 (1990).

[7] O. Gühne, G. Toth, P. Hyllus, and H. J. Briegel, Phys. Rev. Lett. 95, 120405 (2005); A. Cabello and P. Moreno, ibid. 99, 220402 (2007); A. Cabello, O. Guhne, and D. Rodriguez, Phys. Rev. A 77, 062106 (2008).

[8] P. Walther, M. Aspelmeyer, K. J. Resch, and A. Zeilinger, Phys. Rev. Lett. 95, 020403 (2005); N. Kiesel, C. Schmid, U. Weber, G. Toth, O. Guhne, R. Ursin, and H. Weinfurter, ibid. 95, 210502 (2005).

[9] M. Barbieri, F. DeMartini, P. Mataloni, G. Vallone, and A. Cabello, Phys. Rev. Lett. 97, 140407 (2006); R. Ceccarelli, G. Vallone, F. DeMartini, P. Mataloni, and A. Cabello, ibid. 103, 160401 (2009).

[10] Z. Zhao, T. Yang, Y. A. Chen, A. N. Zhang, M. Zukowski, and J. W. Pan, Phys. Rev. Lett. 91, 180401 (2003).

[11] P. Walther, K. J. Resch, T. Rudolph, E. Schenck, H. Weinfurter, V. Vedral, M. Aspelmeyer, and A. Zeilinger, Nature 434, 169 (2005).

[12] N. Kiesel, C. Schmid, U. Weber, G. Toth, O. Guhne, R. Ursin, and H. Weinfurter, Phys. Rev. Lett. 95, 210502 (2005).

[13] R. Prevedel, P. Walther, F. Tiefenbacher, P. Böhi, R. Kaltenbaek, T. Jennewein, and A. Zeilinger, Nature 445, 65 (2007).

[14] K. Chen, C. M. Li, Q. Zhang, Y. A. Chen, A. Goebel, S. Chen, A. Mair, and J. W. Pan, Phys. Rev. Lett. 99, 120503 (2007).

[15] G. Vallone, E. Pomarico, P. Mataloni, F. DeMartini, and V. Berardi, Phys. Rev. Lett. 98, 180502 (2007).

[16] L. Neves, G. Lima, A. Delgado, and C. Saavedra, Phys. Rev. A 80, 042322 (2009).

[17] Y. Tokunaga, S. Kuwashiro, T. Yamamoto, M. Koashi, and N. Imoto, Phys. Rev. Lett. 100, 210501 (2008).

[18] A. Mair, A. Vaziri, G. Weihs, and A. Zeilinger, Nature 412, 313 (2001).

[19] C. Cinelli, M. Barbieri, R. Perris, P. Mataloni, and F. DeMartini, Phys. Rev. Lett. 95, 240405 (2005).
[20] M. Barbieri, C. Cinelli, P. Mataloni, and F. DeMartini, Phys. Rev. A 72, 052110 (2005).

[21] J. T. Barreiro, N. K. Langford, N. A. Peters, and P. G. Kwiat, Phys. Rev. Lett. 95, 260501 (2005).

[22] C. Schuck, G. Huber, C. Kurtsiefer, and H. Weinfurter, Phys. Rev. Lett. 96, 190501 (2006).

[23] H. S. Park, J. Cho, J. Y. Lee, D. Lee, and S. Choi, Opt. Expr. 15, 17960 (2007).

[24] B. P. Lanyon, M. Barbieri, M. P. Almeida, T. Jennewein, T. C. Ralph, K. J. Resch, G. J. Pryde, J. L. O’Brien, A. Gilchrist, and A. G. White, Nature Phys. 5, 134 (2009).

[25] G. Vallone, R. Ceccarelli, F. DeMartini, and P. Mataloni, Phys. Rev. A 79, 030301(R) (2009).

[26] R. Ceccarelli, G. Vallone, F. DeMartini, P. Mataloni, and A. Cabello, Phys. Rev. Lett. 103, 160401 (2009).

[27] B. Dayan, Y. Bromberg, I. Afek, and Y. Silberberg, Phys. Rev. A 75, 043804 (2007)

[28] G. Lima, A. Vargas, L. Neves, R. Guzmán, and C. Saavedra, Opt. Express 17, 10688 (2009).

[29] E. Yao, S. Franke-Arnold, J. Courtial, M. J. Padgett, and S. M. Barnett, Opt. Express 14, 13089 (2006).

[30] R. Rangarajan, M. Goggin, and P. Kwiat, Opt. Express 17, 18920 (2009).

[31] P. G. Kwiat, E. Waks, A. G. White, I. Appelbaum, and P. H. Eberhard, Phys. Rev. A 60, R773 (1999).

[32] S. Cialdi, F. Castelli, I. Boscolo, and M. G. Paris, Appl. Opt. 47, 1832 (2008).

[33] S. Cialdi, F. Castelli, and M. G. A. Paris, J. Mod. Opt. 56, 215 (2009).

[34] M. FrancaSantos, P. Milman, A. Z. Khoury, and P. H. SoutoRibeiro, Phys. Rev. A 64, 023804 (2001).

[35] D. P. Caetano, P. H. Souto Ribeiro, J. T. C. Pardal, and A. Z. Khoury, Phys. Rev. A 68, 023805 (2003).

[36] M. N. O'Sullivan-Hale, I. A. Khan, R. W. Boyd, and J. C. Howell, Phys. Rev. Lett. 94, 220501 (2005).

[37] K. Banaszek, G. M. DAriano, M. G. A. Paris, and M. F. Sacchi, Phys. Rev. A 61, 010304(R) (1999).

[38] D. F. V. James, P. G. Kwiat, W. J. Munro, and A. G. White, Phys. Rev. A 64, 052312 (2001).

[39] G. M. D’Ariano, L. Maccone, and M. G. A. Paris, J. Phys. A 34, 93 (2001).

[40] G. Brida, I. P. Degiovanni, A. Florio, M. Genovese, P. Giorda, A. Meda, M. G. A. Paris, and A. Shurupov, Phys. Rev. Lett. 104, 100501 (2010). 